\title{
Clinical validation of a combinatorial PharmAcogeNomic approach in major Depressive disorder: an Observational prospective RAndomized, participant and rater-blinded, controlled trial (PANDORA trial)
}

Alessandra Minelli ${ }^{1,2+}$, Stefano Barlati ${ }^{3,4+}$, Erika Vitali ${ }^{1,2}$, Stefano Bignotti ${ }^{5}$, Vincenzo Dattilo², Giovanni Battista Tura ${ }^{5}$, Elisabetta Maffioletti ${ }^{1}$, Edoardo Giacopuzzi ${ }^{2}$, Vincenza Santoro ${ }^{6}$, Giulia Perusi ${ }^{3}$, Chiara Cobelli ${ }^{5}$, Chiara Magri ${ }^{1}$, Silvia Bonizzato ${ }^{3}$, Luisella Bocchio-Chiavetto ${ }^{2,7}$, Edoardo Spina ${ }^{6}$, Antonio Vita ${ }^{3,4}$ and Massimo Gennarelli ${ }^{1,2^{*}}$ (D)

\begin{abstract}
Background: Major depressive disorder (MDD) is a common, chronic, debilitating mood disorder that causes serious functional impairment and significantly decreased quality of life. Pharmacotherapy represents the first-line treatment option; however, only approximately one third of patients respond to the first treatment because of the ineffectiveness or side effects of antidepressants. Precision medicine in psychiatry might offer clinicians the possibility to tailor treatment according to the best possible evidence of efficacy and tolerability for each subject. In this context, our study aims to carry out a clinical validation of a combinatorial pharmacogenomics (PGX) test in an Italian MDD patient cohort with advocacy license independence.

Methods: Our study is a prospective participant- and rater-blinded, randomized, controlled clinical observational trial enrolling 300 MDD patients who are referred to psychiatric services to receive a new antidepressant due to the failure of their current treatment and/or the onset of adverse effects. Eligible participants are randomized to the TGTG group (Treated with Genetic Test Guide) or TAU group (Treated as Usual). For all subjects, DNA is collected with a buccal brush. The primary outcome is the reduction in depressive symptomatology. The secondary outcomes involve a range of scales that assess MDD symptoms and social functioning outcomes. The assessment is performed at four timepoints: baseline and 4, 8, and 12 weeks.
\end{abstract}

\footnotetext{
* Correspondence: gennarelli@fatebenefratelli.eu

${ }^{+}$Alessandra Minelli and Stefano Barlati contributed equally to this work.

'Department of Molecular and Translational Medicine, University of Brescia, Brescia, Italy

${ }^{2}$ Genetics Unit, IRCCS Istituto Centro San Giovanni di Dio Fatebenefratelli, Via Pilastroni, 4, 25125 Brescia, Italy

Full list of author information is available at the end of the article
}

(c) The Author(s). 2021 Open Access This article is licensed under a Creative Commons Attribution 4.0 International License, which permits use, sharing, adaptation, distribution and reproduction in any medium or format, as long as you give appropriate credit to the original author(s) and the source, provide a link to the Creative Commons licence, and indicate if changes were made. The images or other third party material in this article are included in the article's Creative Commons licence, unless indicated otherwise in a credit line to the material. If material is not included in the article's Creative Commons licence and your intended use is not permitted by statutory regulation or exceeds the permitted use, you will need to obtain permission directly from the copyright holder. To view a copy of this licence, visit http://creativecommons.org/licenses/by/4.0/. The Creative Commons Public Domain Dedication waiver (http://creativecommons.org/publicdomain/zero/1.0/) applies to the data made available in this article, unless otherwise stated in a credit line to the data. 
Discussion: This project represents the first randomized controlled clinical trial to investigate whether a noncommercial PGx test improves outcomes in an MDD naturalistic cohort. Moreover, the identification of new genetic variants associated with non-response or side effects will improve the efficacy of the test, leading to further costsaving.

Trial registration number: ClinicalTrials.gov NCT04615234. Registered on November 4, 2020.

Keywords: Major depressive disorder, Depression, Pharmacogenetic testing, Randomized controlled clinical, Precision medicine, Antidepressant response, Efficacy

\section{Background}

Major depressive disorder (MDD) is the most common psychiatric disease worldwide and represents a leading cause of years lived with disability, resulting in a substantial socio-economic impact [1]. The main goal of treating MDD is to achieve remission and to maintain the therapeutic effects over time. Despite the availability of different classes of antidepressant drugs (ADs), the success of pharmacological treatment is still unsatisfactory, and matching a patient to his/her optimal treatment generally requires multiple trials of different treatments, with the sobering observation that the more treatments that are tried without success, the less likely a successful outcome is. Only approximately one third of patients achieve remission after the first treatment course, while another approximately one third develop treatment-resistant depression (TRD) $[2,3]$. The high percentage of treatment failure or incomplete remission could be a consequence of intrinsic biological and environmental heterogeneity among MDD patients [4, 5], suggesting that biomarkers of the response to ADs would be useful for clinicians to guide treatment at the individual level. In this context, pharmacogenomics (PGx) testing has the potential to reduce antidepressant discontinuation due to side effects and increase efficacy.

Recently, assay-guided treatment has shown promising results. Several observational and randomized controlled trials (RCTs) have been conducted to investigate the impact of pharmacogenetics or pharmacogenomics testing on antidepressant outcome in MDD patients with interesting results $[6,7]$.

This article presents the protocol for the RCT and is written to comply with the recommended SPIRIT guidelines for RCT protocols (Additional file 1).

\section{Aim of the study}

The study was designed as an observational, prospective participant- and rater-blinded randomized, controlled trial (shown in Figs. 1 and 2) to evaluate the clinical efficacy of a combinatorial PGx test to guide clinician' treatment decision-making in a naturalistic setting. This study will be conducted in an Italian MDD patient cohort with advocacy license independence. In particular, the main objective of the study is to assess the role of a PGx test in improving the response rate and leading to a greater amelioration of depressive symptoms in MDD patients. The secondary objective is to evaluate the use of PGx in decreasing the side effects of antidepressants. Moreover, the study aims to provide data about the use of the PGx test in decreasing depressive-related symptomatology (such as anxiety symptoms and functionality) and the possible influence of vulnerability factors, such as early and recent stressful events, on the main outcomes.

\section{Methods \\ Eligibility criteria}

Three hundred MDD out-patients are voluntarily enrolling in the study. Patients are referred to psychiatric services (University Department of Mental Health, Spedali

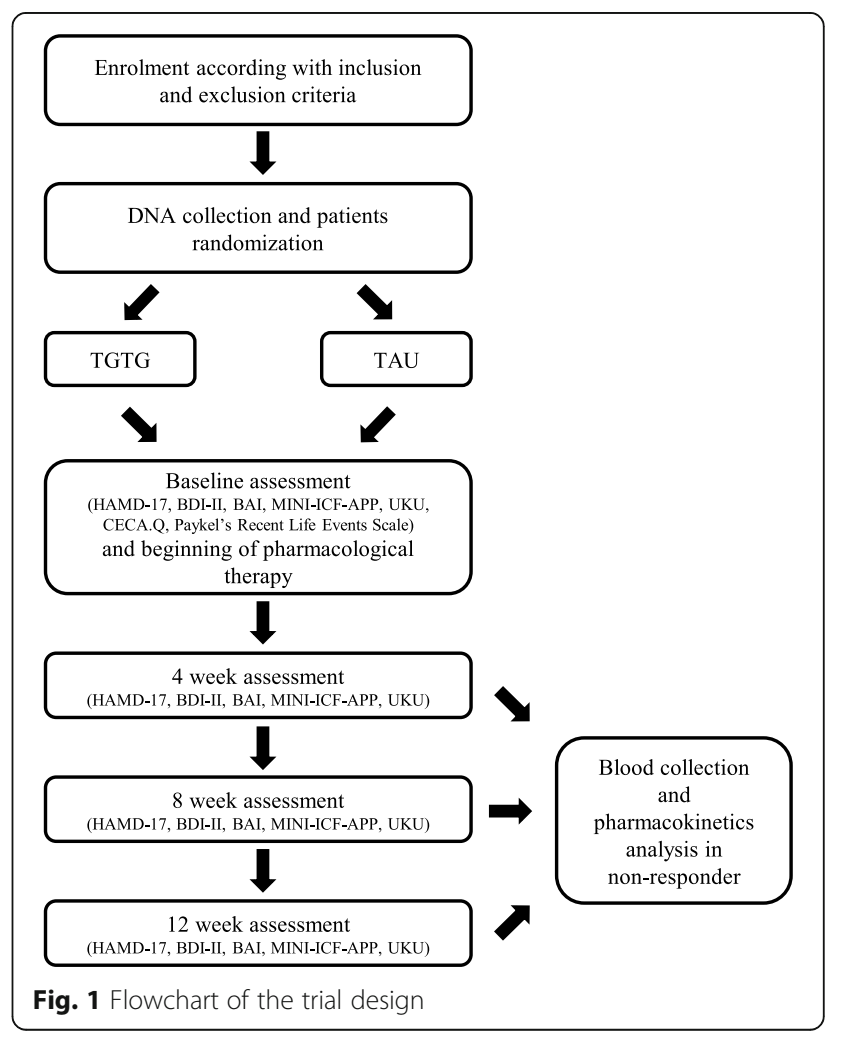




\begin{tabular}{|c|c|c|c|c|c|c|}
\hline & \multicolumn{6}{|c|}{ STUDY PERIOD } \\
\hline \multirow[b]{2}{*}{ TIMEPOINT } & \multirow[t]{2}{*}{ Enrolment } & \multirow[t]{2}{*}{ Allocation } & \multicolumn{4}{|c|}{ Timepoints } \\
\hline & & & TO & $T 4$ & $T 8$ & $T 12$ \\
\hline \multicolumn{7}{|l|}{ ENROLMENT: } \\
\hline $\begin{array}{r}\text { Eligibility screening } \\
\text { (according with } \\
\text { diagnostic inclusion } \\
\text { and exclusion } \\
\text { criteria) }\end{array}$ & $\mathrm{x}$ & & & & & \\
\hline \multirow{2}{*}{$\begin{array}{r}\text { Written informed } \\
\text { consent signature } \\
\text { DNA collection } \\
\text { through buccal } \\
\text { brush }\end{array}$} & $\mathrm{x}$ & & & & & \\
\hline & $x$ & & & & & \\
\hline \multicolumn{7}{|l|}{$\begin{array}{r}\text { Random allocation } \\
\text { (TGTG, TAU) }\end{array}$} \\
\hline \multicolumn{7}{|l|}{ INTERVENTIONS: } \\
\hline \multicolumn{7}{|l|}{$\begin{array}{c}\text { Administration of } \\
\text { new AD treatment }\end{array}$} \\
\hline $\begin{array}{r}\text { Pharmacokinetic } \\
\text { analysis in both } \\
\text { TGTG and TAU non- } \\
\text { responder patients }\end{array}$ & & & & $\mathrm{x}$ & $x$ & $\mathrm{x}$ \\
\hline \multicolumn{7}{|l|}{ ASSESSMENTS: } \\
\hline \multirow{2}{*}{\multicolumn{7}{|c|}{$\begin{array}{r}\text { HAMD-17, BDI-II, } \\
\text { BAI, MINI-ICF-APP, } \\
\text { UKU Side Effects } \\
\text { Rating Scale } \\
\\
\text { CECA.Q, Paykel's } \\
\text { Recent Life Events } \\
\text { Scale } \\
\end{array}$}} \\
\hline & & & & & & \\
\hline \multicolumn{7}{|l|}{ OUTCOMES: } \\
\hline Primary outcomes & & & & & $x$ & \\
\hline Secondary outcomes & & & & $x$ & $x$ & $x$ \\
\hline Tertiary outcomes & & & & $x$ & $\mathrm{x}$ & $x$ \\
\hline
\end{tabular}

Fig. 2 Schedule of enrolment, interventions, assessments, and outcomes of the PANDORA trial

Civili Hospital, and IRCCS Istituto Centro San Giovanni di Dio Fatebenefratelli - Brescia, Italy) to receive a new $\mathrm{AD}$ due to the failure of their current treatment and/or the onset of adverse effects. The diagnostic inclusion criteria are as follows: a current diagnosis of unipolar depression according to the Diagnostic and Statistical Manual of Mental Disorders-5 (DSM-5) [8] classification system criteria with a Hamilton Depression Rating Scale (HAMD-17) [9] score $\geq 14$, age range 18-65 years, and Caucasian ethnicity. The exclusion criteria are cognitive impairment (Mini Mental State Examination MMSE < 24) [10]; neurological disorders; diagnosis of MDD with psychotic features, bipolar I and II disorders, schizophrenia spectrum and other psychotic disorders, obsessivecompulsive disorder, post-traumatic stress disorder, alcohol and substance abuse in the last 3 months; comorbidity with personality disorders (cluster A and/or B); pregnancy; and comorbidity with other severe medical illness. Diagnoses are confirmed using the Italian version of the Structural Clinical Interview for DSM-5 disorders (SCID-5-CV) [11] and the Structural Clinical Interview for personality disorders (SCID-5-PD) [12]. After the enrolment of MDD patients in accordance with the inclusion and exclusion criteria and after obtaining written informed consent, for all patients, DNA is collected through a buccal brush.

\section{Randomization and concealment}

Eligible patients are randomized to the Treated with Genetic Test Guide (TGTG) group or Treated as Usual (TAU) group. The allocation to the TGTG or TAU group is performed with simple randomization software 
(http://glimmpse.samplesizeshop.org/) by the project manager who is not involved in either the evaluation or the treatment. The allocation of the patients is communicated directly from the project manager (AM) to the prescriber. None of the other people involved in the projects are informed and/or have access to the allocation information. The randomized allocation sequence of the trial participants is conserved in a double file protected by a double password owned by the PI (MG) and the project manager (AM).

\section{Assessments}

The clinical assessment is carried out at 4 timepoints: baseline (T0), after 4 (T4), 8 (T8), and 12 (T12) weeks of $\mathrm{AD}$ treatment. The evaluations are performed using the HAMD-17, the Beck Depression Inventory II (BDI-II) [13]; the Beck Anxiety Inventory (BAI) [14], to evaluate the clinical efficacy of the therapy; the MINI-ICF-APP [15] to monitor changes in psychosocial functioning; and the UKU (Udvalg for Kliniske Undersogelser) Side Effects Rating Scale [16] to observe the adverse events. Moreover, the Childhood Experience of Care and Abuse Questionnaire (CECA.Q) [17] and the Paykel's Recent Life Events Scale [18] are assessed only at T0.

\section{Blinding}

All the participants are evaluated by assessors who are not otherwise involved, pharmacological treatment, or patient allocation. The treating physicians are unblinded. Neither the assessors nor prescribers are involved in the data analysis.

\section{Pharmacogenetic report and intervention procedure}

The clinicians of the patients in the TGTG group receive the PGx test report within $48 \mathrm{~h}$, and all the participants start their new treatment within $72 \mathrm{~h}$. The PGx test results for the subjects in the TAU are provided to the prescriber once all week 12 visit procedures are completed. AD monotherapy is mandatory, with the exception of the association of benzodiazepines and/or hypnotics when necessary. If the patients need another change in antidepressant monotherapy, they stay in the study protocol, and the changes are annotated. If the patients need a combination and/or augmentation treatment with antipsychotics and/or mood stabilizers, they are excluded from the study.

\section{Outcomes}

The primary outcome is symptom improvement at week 8 , as measured by the percent change in the HAMD-17 score from baseline, between the two groups.

Secondary outcomes include response and remission rates at 4,8 , and 12 weeks according to the HAMD- 17 .
Tertiary outcomes include (1) changes in scores of depressive symptoms at 4,8 , and 12 weeks compared with baseline according to the BDI-II; (2) response and remission rate at 4,8 , and 12 weeks according to BDI-II; (3) changes in scores of anxiety symptoms at 4, 8, and 12 weeks compared with baseline as measured by the BAI; (4) changes in scores of psychosocial functioning at 8 and 12 weeks compared with baseline as measured by the MINI-ICF-APP; and (5) side effects at 4,8 , and 12 weeks as assessed by the UKU Side Effect Rating Scale.

The response is defined as a $\geq 50 \%$ decrease in the assessment of interest (HAMD-17, BDI-II) at weeks 4, 8, and 12 compared with the baseline. Remission is defined as a score of $\leq 7$ for HAMD- 17 and $\leq 9$ for BDI-II.

\section{Genotyping}

Buccal cell samples are collected by FLOQSwab hDNA Free buccal brushes (Copan Brescia, Italy). Genomic DNA extraction is performed with a Quick DNA Miniprep plus Kit (ZymoResearch, California, USA) according to the manufacturer's instructions.

In our trial, we investigate the genetic variants reported in the PharmGKB database (www.pharmgkb.org) with a clinical annotation of evidence of association with $\mathrm{AD}$ response classified as levels $1 \mathrm{a}, 1 \mathrm{~b}, 2 \mathrm{a}$, and $2 \mathrm{~b}$.

Our PGx test includes 31 genetic variants. Thirty single-nucleotide polymorphisms (SNPs) are genotyped with customized TaqMan OpenArray plates on a QuantStudio 12K Flex Real-Time PCR System (Applied Biosystems, Foster City, California, USA) according to the manufacturer's instructions (15 in CYP2D6, 10 in CYP2C19, and four in MC4R, FKBP5, $H T R 1 A$, and HTR2A). The data are analyzed with the Genotyping application on Thermo Fisher Cloud. Moreover, copy number variation $(\mathrm{CNV})$ of the CYP2D6 gene is evaluated using the TaqMan Copy Number Assay mix specific for CYP2D6 exon 9 (Assay ID: Hs00010001_cn) according to the manufacturer's instructions and with a $C_{\mathrm{T}}$ threshold of 0.2 , and the analysis is performed with CopyCaller Software (Applied Biosystems). Using AlleleTyper Software (Life Technologies, California, USA), we integrate the SNP genotyping results with the copy number information for the CYP2D6 gene to obtain all the eventual CYP2D6 and CYP2C19 diplotypes. Diplotypes with higher allele frequencies in the European population are selected if it cannot be determined with certainty. The translation is based on the translation table obtained from the PharmGKB database.

Participants are also genotyped for the 5-HTTLPR (short/long allele) in the SLC6A4 gene by PCR amplification of the relevant genomic location using the KAPA HiFi 
HotStart PCR Kit (Roche Diagnostics, Basel, Switzerland) and the following primers: 5'-ATGCCAGCACCTAACCCCTAATGT-3' (forward) and 5'-GGACCGCAAGGTGGGCGGGA-3' (reverse). The genotype is then determined by electrophoresis and visualization of the amplified products on a $2 \%$ agarose gel.

\section{Pharmacogenomic (PGx) test report}

Considering the resulting genetic profile, a personalized PGx report is generated. The diplotypes obtained for the CYP2D6 and CYP2C19 genes are associated with the matching metabolizer phenotypes: ultra-rapid, normal, intermediate, and poor for CYP2D6, and ultra-rapid, rapid, intermediate, poor, normal, likely intermediate, and likely poor for CYP2C19.

According to both the Clinical Pharmacogenetics Implementation Consortium (CPIC) and the Dutch Pharmacogenetics Working Group (DPWG) guidelines, the report places the most ADs widely used in Italy into three recommended categories: (1) "use as directed" (labeled "green"), (2) "use with caution" (labeled "yellow"), and (3) "use with extreme caution" (labeled "red") (see Fig. 3 for an example of a report for one patient). In addition, drug details associated with each medication in the yellow or red categories are provided. The current $\mathrm{AD}$ is excluded from the report to avoid clinician bias in decision-making. The clinicians have access to the PGx report through an interactive web interface.

Before study initiation, training was provided to all participating investigators on the interpretation of genetic testing results and on the relevance of each genetic variant to pharmacotherapy.

\section{Pharmacokinetic analysis}

Pharmacokinetic evaluation is conducted at weeks 4, 8, and 12 in the TAU group and the non-responder patients in the TGTG group. Serum concentrations of the ADs and their metabolites are measured at a steadystate on the same day of clinical evaluation. Blood samples for pharmacokinetic analysis are drawn at 8 a.m. before the morning AD dose. Determination of the serum concentrations of the ADs and their metabolites are performed by using high-performance liquid chromatography procedures.

\section{Further pharmacogenomics analysis}

To better elucidate the molecular mechanisms underlying the response to the ADs, for all non-responder patients in our trial, further PGx analysis will be carried out. In particular, we will first investigate, always through a TaqMan OpenArray approach, further genetic variants known to be involved in the response to ADs or their side effects that are already described in literature studies and in the PharmGKB database. Moreover, for those patients (belonging to both the TGTG and TAU groups) who do not benefit from the assigned treatment, sequencing of all the genes associated with both $\mathrm{AD}$ response and susceptibility to side effects will be conducted with the aim of identifying novel rare functional variants.

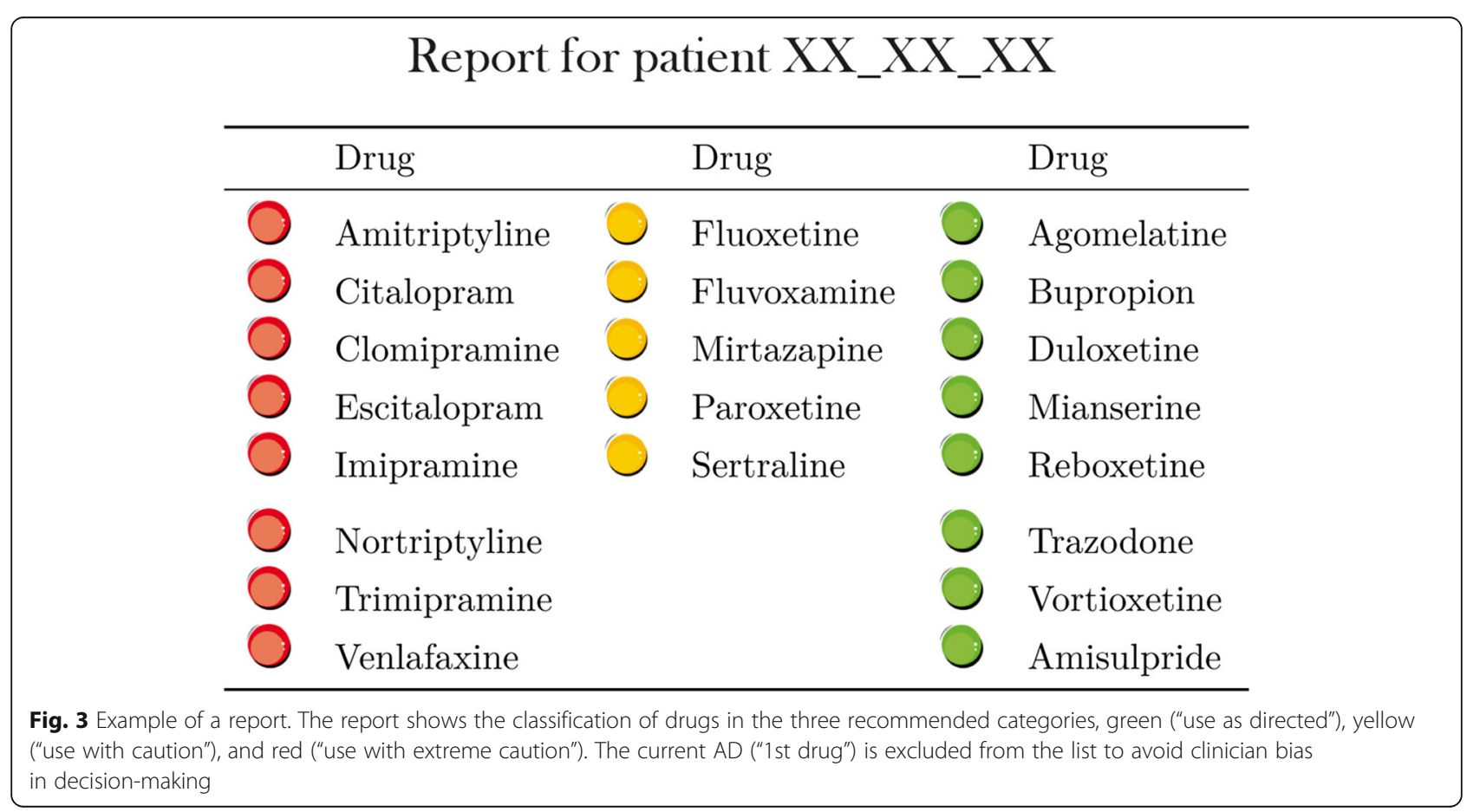




\section{Sample size}

Based on previous PGx studies performed on MDD patient cohorts $[19,20]$, the sample size needed to detect a significant reduction in symptom scores was calculated. The analysis revealed that a sample size of 225 participants ( 112 per group) is required to observe a 15\% reduction in symptom scores with a common standard deviation of $45 \%$, alpha $=0.05$, and power $=80 \%$. Assuming an estimated drop-out rate of $20-25 \%$, as already observed in follow-up studies on MDD [21], a total sample size of 300 participants will be enrolled in this study.

\section{Data management}

The data management process is the responsibility of the project coordinator. Both clinical and biological data collection, analysis, storage, security, and sharing are consistent with the standard operating procedures that ensure patient pseudonymization.

\section{Baseline demographic and clinical features}

Age (years) and mean (SD); gender (\%F); education (years) and mean (SD); race (\%); smokers (\%); body mass index (BMI) and mean (SD); age of onset (years) and mean (SD); depression category; moderate, severe, or very severe (\%); recurrence (\%); presence of psychotic symptoms (\%); comorbidity with personality disorders congruent with inclusion/exclusion criteria (\%); comorbidity with anxiety disorders (\%); presence of psychiatric disorders among the first-degree relatives (\%); HAMD17 at baseline and mean (SD); BDI-II at baseline and mean (SD); BAI-II at baseline and mean (SD); mini-ICFAPP at baseline and mean (SD); MMSE at baseline and mean (SD); UKU at baseline and mean (SD); previous failed adequate treatment; CECA-Q mother antipathy (hostility, coldness) (\%); CECA-Q father antipathy (hostility, coldness) (\%); CECA-Q mother neglect (\%); CECA-Q father neglect (\%); CECA-Q physical abuse mother (\%); CECA-Q physical abuse father (\%); CECA$\mathrm{Q}$ sexual abuse (\%); and Paykel score and means (SD).

\section{Statistical analysis}

Analysis of variance (ANOVA) or the Kruskal-Wallis and Mann-Whitney $U$ nonparametric tests and the chisquared test will be used to analyze differences in continuous and categorical variables, respectively, between groups. Pearson's correlation analysis will be used to evaluate bivariate correlations. Parametric and nonparametric tests will be used to meet relative assumptions (i.e., distribution, sample size).

To pursue our primary outcome, the analyses will be performed for patients who complete the study through week 8 . The analysis will be performed using mixedeffects models with repeated measures (MMRM) to examine the effect of time and group (TGTG vs. TAU) on the reduction in the HAMD-17 score. The model includes the fixed effect continuous factor baseline HAMD-17 and fixed effect categorical factors, which are the treatment group (TGTG and TAU; 2 levels), visit (weeks 4 and 8; 2 levels), and treatment $\mathrm{x}$ visit interaction. The mean changes in the HAMD-17 at week 8 in the TGTG and TAU groups will be estimated and tested utilizing the LS means from the treatment $\mathrm{x}$ visit interaction in the MMRM model. The primary analysis will test the difference (contrast) between the week 8 least squares (LS) means at a two-sided significance of 0.05 . To achieve the secondary outcome, the generalized linear mixed model will be used for response and remission analyses. The analyses of the tertiary outcomes will be performed for exploratory purposes with the same statistical models. Comparisons between the TGTG and TAU means at all timepoints of evaluation will also be performed for descriptive purposes. Finally, an intention-totreat (ITT) analysis will be performed for all the patients who undergo at least one post-treatment assessment for efficacy during the study. The last observation will be carried forward on the HAMD-17. All the statistical analyses will be performed using $\mathrm{R}$ Statistical Software ( $\mathrm{R}$ Foundation for Statistical Computing, Vienna, Austria).

\section{Discussion}

Genetic variants play important roles in the responses of MDD patients to ADs, explaining approximately $40 \%$ of pharmacological treatment outcomes [22, 23]. Based on this evidence, different studies have been performed to evaluate the utility of treatment guided by a PGx test, which investigates the possible response to ADs according to the genetic background of the patient. These studies revealed a better outcome in patients treated with guided care, both in terms of response rates and in terms of a decrease in reported side effects, confirming the utility of the PGx test in the treatment of MDD [24]. However, few of these studies performed are RCTs, and consequently, further ones are needed to increase the understanding regarding the clinical utility of such tests that include both genetic profile characterization and clinical assessment symptomatology.

In this context, the aim of our study is to evaluate the clinical utility of a combinatorial PGx test by performing an observational, prospective, participant -and raterblinded, randomized, controlled trial in an Italian MDD patient cohort with advocacy license independence.

The use of a tool based on a combinatorial approach provides clinicians with more complete information about a patient's response to drugs. Indeed, although individual gene test panels provide information about the effects of an individual gene on each investigated drug, the combinatorial PGx test considers the simultaneous effects of different genes on drug pharmacokinetics and 
pharmacodynamics, providing information that is more accurate and rapidly applicable to clinician practice [25].

The longitudinal evaluation of outcomes at four different timepoints allows us to assess the efficacy of the PGx test to suggest a therapy that could be efficient in the long term. Moreover, the application of a wide range of rating scales provides a complete view of outcomes, both in terms of symptom improvement and the development of adverse effects, and allows us to study the impact of the PGx test on the different symptom phenotypes of the disease. Moreover, the high number of variants investigated along with a wide range of clinical symptoms characterization that will be performed, allowing us to evaluate the possible association between endophenotypes and specific symptom improvement.

In addition, this trial will provide further information about the genetic variance and the distribution of phenotype metabolizers in an Italian sample of patients with MDD, increasing the amount of data available for the scientific community. Furthermore, in nonresponder patients, an in-depth investigation of further genetic variants implicated in treatment outcomes will contribute to enriching the knowledge about the molecular mechanisms underlying the response to ADs.

Finally, the periodical update of the PGx report software will allow us to provide indications for each $\mathrm{AD}$ based on more recent versions of the CPIC and DPWG guidelines.

There are some possible limitations of this study. First, as in all the other PG test validation studies, the treating clinician is not blinded to the study arm. This is necessary for ethical issues related to mandating prescribed medications to blind clinicians. To mitigate this limitation, raters and patients were blinded to the study arm until week 12 . Second, the majority of the cohort that will be recruited will be limited to the psychiatric services of the Lombardy region. This could affect the project both in terms of impact on the disease and treatment outcome due to the local organization of mental health services and in terms of ethnicities represented in the cohort. This may limit the generalizability of the results to a wider population of MDD patients.

\section{Trial status}

This article is based on the study protocol version 1.2 of August 8, 2018. The study protocol was posted to ClinicalTrials.gov as NCT04615234 (Registration date: November 4, 2020). The recruitment of patients started on February 1, 2020, and will be completed after approximately 30 months. Due to the COVID-19 emergency, the period required for the completion of the study will be longer.

\section{Abbreviations}

MDD: Major depressive disorder; ADs: Antidepressant drugs; TRD: Treatmentresistant depression; PGx: Pharmacogenomics; RCTs: Randomized controlled trials; DSM-5: Diagnostic and Statistical Manual of Mental Disorders-5; HAMD17: Hamilton Depression Rating Scale; MMSE: Mini Mental State Examination; SCID-5-CV: Structural Clinical Interview for DSM-5 disorders; SCID-5-

PD: Structural Clinical Interview for personality disorders; TGTG: Treated with Genetic Test Guide; TAU: Treated As Usual; BDI-II: Beck Depression Inventory II; BAI: Beck Anxiety Inventory; UKU: Udvalg for Kliniske Undersogelser;

CECA.Q: Childhood Experience of Care and Abuse Questionnaire;

PharmGKB: Pharmacogenomics Knowledge Base; SNPs: Single-nucleotide polymorphisms; CNV: Copy number variation; CPIC: Clinical

Pharmacogenetics Implementation Consortium; DPWG: Dutch

Pharmacogenetics Working Group

\section{Supplementary Information}

The online version contains supplementary material available at https:/doi. org/10.1186/s13063-021-05775-8.

Additional file 1. SPIRIT 2013 Checklist: Recommended items to address in a clinical trial protocol and related documents*.

\section{Acknowledgements}

We thank Copan Italia to have gently provided buccal brush.

\section{Biological specimens}

The biological samples for genetic analysis are stored at the IRCCS Istituto Centro San Giovanni di Dio Fatebenefratelli, and all laboratory evaluations are performed at the Genetics Unit, IRCCS Istituto Centro San Giovanni di Dio Fatebenefratelli, Brescia, Italy, and at the Clinical and Experimental Medicine, University of Messina, Messina, Italy.

\section{Authors' contributions}

$A M, S B, E S, A V$, and $M G$ made a significant contribution to the conception of the study and implementation of the protocol. AM, SB, SBi, GBT, ES, AV, and MG participated in the design of the study. SB, SBi, GBT, GP, CC, SBo, and AV following patient recruitment. $\mathrm{AM}, \mathrm{SB}$, and $\mathrm{EV}$ participated in the writing process of the first draft of the manuscript. ES, AV, and MG made a revision to the manuscript. AM, EV, and VD performed the statistical analysis and data management. EV, VD, EM, and VS performed genetic and biological analysis. $\mathrm{EV}, \mathrm{VD}, \mathrm{EG}$, and $\mathrm{CM}$ developed the software for producing the pharmacogenetic report. The authors read and approved the final manuscript. MG is the PI of the project.

\section{Funding}

This study was funded by a grant provided by the Italian Ministry of Health (Ricerca Finalizzata RF-2016-02361697).

\section{Availability of data and materials}

For processing the data, we use the pseudonymization procedure so that the same data can no longer be attributed to a specific subject without the use of additional information. The correspondence between the code and the personal information of the subject is contained in a separate file protected by passwords. Only the PI (MG) and the project manager (AM) have access to the final dataset. Patient data as well as the statistical analyses are stored on a local server of the IRCCS Istituto Centro San Giovanni di Dio Fatebenefratelli respective international data security standards.

\section{Declarations}

\section{Ethics approval and consent to participate}

The study was approved by the Local Ethics Committees (CEIOC IRCCS Istituto Centro San Giovanni di Dio Fatebenefratelli, Brescia N: 43/2018 and Ethics Committee of ASST Spedali Civili of Brescia N: NP 3347), and all patients signed a written informed consent to participate after receiving a comprehensive explanation of the study procedures and goals, according to the institutional guidelines of the local Ethics Committees. 


\section{Consent for publication}

Not applicable since this manuscript does not contain any individual's data in any form.

\section{Competing interests}

The authors declare that they have no competing interests.

\section{Author details}

'Department of Molecular and Translational Medicine, University of Brescia, Brescia, Italy. ${ }^{2}$ Genetics Unit, IRCCS Istituto Centro San Giovanni di Dio Fatebenefratelli, Via Pilastroni, 4, 25125 Brescia, Italy. ${ }^{3}$ Department of Mental Health and Addiction Services, ASST Spedali Civili of Brescia, Brescia, Italy. ${ }^{4}$ Department of Clinical and Experimental Sciences, University of Brescia, Brescia, Italy. ${ }^{5}$ Psychiatry Unit, IRCCS Istituto Centro San Giovanni di Dio Fatebenefratelli, Brescia, Italy. ${ }^{6}$ Clinical and Experimental Medicine, University of Messina, Messina, Italy. ${ }^{7}$ Faculty of Psychology, eCampus University, Novedrate, Como, Italy.

Received: 15 April 2021 Accepted: 29 October 2021

Published online: 11 December 2021

\section{References}

1. Hasin DS, Sarvet AL, Meyers JL, Saha TD, Ruan WJ, Stohl M, et al. Epidemiology of adult DSM-5 major depressive disorder and its specifiers in the United States. JAMA Psychiatry. 2018;75:336-46 Available from: http:// www.ncbi.nIm.nih.gov/pubmed/29450462.

2. Souery D, Serretti A, Calati R, Oswald P, Massat I, Konstantinidis A, et al. Switching antidepressant class does not improve response or remission in treatment-resistant depression. J Clin Psychopharmacol. 2011;31:512-6 Available from: http://www.ncbi.nlm.nih.gov/pubmed/21694617.

3. Gaynes BN, Rush AJ, Trivedi MH, Wisniewski SR, Spencer D, Fava M. The STAR*D study: treating depression in the real world. Cleve Clin J Med. 2008; 75:57-66 Available from: http://www.ncbi.nlm.nih.gov/pubmed/18236731.

4. Fabbri C, Serretti A. Genetics of treatment outcomes in major depressive disorder: present and future. Clin Psychopharmacol Neurosci. 2020;18:1-9 Available from: http://www.ncbi.nlm.nih.gov/pubmed/31958900.

5. Gratten J, Wray NR, Keller MC, Visscher PM. Large-scale genomics unveils the genetic architecture of psychiatric disorders. Nat Neurosci. 2014;17:78290 Available from: http://www.ncbi.nlm.nih.gov/pubmed/24866044.

6. Fabbri C, Zohar J, Serretti A. Pharmacogenetic tests to guide drug treatment in depression: comparison of the available testing kits and clinical trials. Prog Neuropsychopharmacol Biol Psychiatry. 2018;86:36-44 Available from: http://www.ncbi.nlm.nih.gov/pubmed/29777729.

7. Bousman CA, Arandjelovic K, Mancuso SG, Eyre HA, Dunlop BW. Pharmacogenetic tests and depressive symptom remission: a meta-analysis of randomized controlled trials. Pharmacogenomics [Internet]. 2019;20:3747 Available from: http://www.ncbi.nlm.nih.gov/pubmed/30520364.

8. APA. Diagnostic and statistical manual of mental disorders. 5th ed. Arlington, VA: American Psychiatric Association; 2013.

9. Fleck MP, Poirier-Littre MF, Guelfi JD, Bourdel MC, Loo H. Factorial structure of the 17-item Hamilton Depression Rating Scale. Acta Psychiatr Scand [Internet]. 1995;92:168-72 Available from: http://www.ncbi.nlm.nih.gov/ pubmed/7484192.

10. Folstein MF, Folstein SE, McHugh PR. "Mini-mental state". A practical method for grading the cognitive state of patients for the clinician. J Psychiatr Res [Internet]. 1975;12:189-98 Available from: http://www.ncbi.nlm. nih.gov/pubmed/1202204.

11. First M, Williams J, Karg R, Spitzer R. Structured clinical interview for DSM-5 disorders, clinician version (SCID-5-CV). Arlington, VA: American Psychiatric Association; 2016.

12. First M, Williams J, Benjamin L, Spitzer R. User's Guide for the SCID-5-PD (structured clinical interview for DSM-5 personality disorder). Arlington, VA: American Psychiatric Association; 2015.

13. Beck AT, Steer R, Brown G. Manual for the beck depression inventory-ll. San Antonio, TX: Psychological Corporation; 1996.

14. Beck AT, Steer R. Beck anxiety inventory manual. San Antonio, TX: Psychological Corporation; 1993.

15. Balestrieri M, Isola M, Bonn R, Tam T, Vio A, Linden M, et al. Validation of the Italian version of Mini-ICF-APP, a short instrument for rating activity and participation restrictions in psychiatric disorders. Epidemiol Psychiatr Sci
[Internet]. 2013;22:81-91 Available from: http://www.ncbi.nlm.nih.gov/ pubmed/22989494.

16. Lingjaerde O, Ahlfors UG, Bech P, Dencker SJ, Elgen K. The UKU side effect rating scale. A new comprehensive rating scale for psychotropic drugs and a cross-sectional study of side effects in neuroleptic-treated patients. Acta Psychiatr Scand Suppl [Internet]. 1987;334:1-100 Available from: http://www. ncbi.nlm.nih.gov/pubmed/2887090.

17. Bifulco A, Bernazzani O, Moran PM, Jacobs C. The childhood experience of care and abuse questionnaire (CECA.Q): validation in a community series. $\mathrm{Br}$ J Clin Psychol [Internet]. 2005;44:563-81 Available from: http://www.ncbi. nlm.nih.gov/pubmed/16368034.

18. Baratta S, Colorio C, Zimmermann-Tansella C. Inter-rater reliability of the Italian version of the Paykel Scale of stressful life events. J Affect Disord [Internet]. 1995;8:279-82 Available from: http://www.ncbi.nlm.nih.gov/ pubmed/3160739.

19. Pérez V, Salavert A, Espadaler J, Tuson M, Saiz-Ruiz J, Sáez-Navarro C, et al. Efficacy of prospective pharmacogenetic testing in the treatment of major depressive disorder: results of a randomized, double-blind clinical trial. BMC Psychiatry [Internet]. 2017;17:250 Available from: http://www.ncbi.nlm.nih. gov/pubmed/28705252.

20. Hall-Flavin DK, Winner JG, Allen JD, Carhart JM, Proctor B, Snyder KA, et al. Utility of integrated pharmacogenomic testing to support the treatment of major depressive disorder in a psychiatric outpatient setting. Pharmacogenet Genomics. 2013;23(10):535-48. https://doi.org/10.1097/FPC. Ob013e3283649b9a.

21. Hans $E$, Hiller W. Effectiveness of and dropout from outpatient cognitive behavioral therapy for adult unipolar depression: a meta-analysis of nonrandomized effectiveness studies. J Consult Clin Psychol. 2013;81(1):7588. https://doi.org/10.1037/a0031080.

22. Tansey KE, Guipponi M, Hu X, Domenici E, Lewis G, Malafosse A, et al. Contribution of common genetic variants to antidepressant response. Biol Psychiatry Elsevier. 2013;73(7):679-82. https://doi.org/10.1016/j.biopsych.2 012.10.030.

23. Corponi F, Fabbri C, Serretti A. Pharmacogenetics and depression: a critical perspective. Psychiatry Investig. 2019;16(9):645-53. https://doi.org/10.30773/ pi.2019.06.16.

24. Brown L, Vranjkovic O, Li J, Yu K, Al Habbab T, Johnson H, et al. The clinical utility of combinatorial pharmacogenomic testing for patients with depression: a meta-analysis. Pharmacogenomics. 2020;21 (8):559-69. https:// doi.org/10.2217/pgs-2019-0157.

25. Winner JG, Dechairo B. Combinatorial versus individual gene pharmacogenomic testing in mental health: a perspective on context and implications on clinical utility. Yale J Biol Med. 2015;88(4):375-82.

\section{Publisher's Note}

Springer Nature remains neutral with regard to jurisdictional claims in published maps and institutional affiliations.

Ready to submit your research? Choose BMC and benefit from

- fast, convenient online submission

- thorough peer review by experienced researchers in your field

- rapid publication on acceptance

- support for research data, including large and complex data types

- gold Open Access which fosters wider collaboration and increased citations

- maximum visibility for your research: over $100 \mathrm{M}$ website views per year

At $\mathrm{BMC}$, research is always in progress.

Learn more biomedcentral.com/submissions 